\title{
BMJ Open Predicting value of white cell count and total bilirubin on clinical outcomes in patients with ST-elevation myocardial infarction following percutaneous coronary intervention: a cohort study
}

\author{
Munire Tuxun, ${ }^{1}$ Qian Zhao, ${ }^{1,2,3}$ Yang Xiang, ${ }^{1}$ Fen Liu, ${ }^{1,2,3}$ Chun-Fang Shan, \\ Xin-Rong Zhou, ${ }^{1,2}$ Ning Song, ${ }^{1}$ Ajiguli Waisiding, ${ }^{1}$ Xue-He Zhang, ${ }^{1}$ \\ Gulandanmu Aihemaiti, ${ }^{1}$ Yi-Ning Yang, ${ }^{1,2,3}$ Xiao-Mei Li (D) ${ }^{1,2,3}$
}

To cite: Tuxun M, Zhao Q, Xiang $\mathrm{Y}$, et al. Predicting value of white cell count and total bilirubin on clinical outcomes in patients with STelevation myocardial infarction following percutaneous coronary intervention: a cohort study. BMJ Open 2020;10:e031227. doi:10.1136/ bmjopen-2019-031227

- Prepublication history and additional material for this paper are available online. To view these files, please visit the journal online (http://dx.doi. org/10.1136/bmjopen-2019031227).

MT and QZ are joint first authors.

Received 13 June 2019 Revised 03 December 2019 Accepted 04 December 2019

Check for updates

(c) Author(s) (or their employer(s)) 2020. Re-use permitted under CC BY-NC. No commercial re-use. See rights and permissions. Published by BMJ.

For numbered affiliations see end of article.

Correspondence to Professor Xiao-Mei Li; lixm505@163.com

Professor Yi-Ning Yang; yangyn5126@163.com

\section{ABSTRACT}

Objectives A combined equation based on white cell count (WCC) and total bilirubin (TB) was assessed for its ability to predict adverse clinical outcomes in patients with acute ST-segment elevation myocardial infarction (STEMI) with primary percutaneous coronary intervention (PCl). Design A single-centre, prospective cohort study. Setting The First Affiliated Hospital of Xinjiang Medical University.

Method A total of 615 patients with STEMI postprimary PCI were enrolled. WCC and TB were collected at admission. Logistic regression was used to determine the combined equation. The primary endpoints were in-hospital mortality and major adverse cardiovascular events (MACE), which composed of cardiac death, cardiac shock, malignant arrhythmia (ventricular tachycardia, ventricular fibrillation), severe cardiac insufficiency, nonfatal myocardial infarction, angina pectoris readmission, severe cardiac insufficiency (cardiac III-IV level), stent restenosis and target vessels revascularisation during the hospitalisation and 36 months follow-up period.

Result 77 patients occurred in MACE during the hospitalisation (17 in-hospital mortality). WCC and TB were taken as an independent variables to make a category of logistic regression analysis of in-hospital MACE, the logistic regression model was: logit $(P)=-8.00+0.265$ WCC+0.077TB, the combination of WCC and TB was more valuable on evaluating the in-hospital mortality (area under the curve $0.804,95 \% \mathrm{Cl} 0.678$ to 0.929 , $\mathrm{p}<0.001)$. Multivariate logistic regression analysis showed that combined detection was an independent risk factor for in-hospital MACE (OR 5.85, 95\% CI 3.425 to 9.990, $\mathrm{p}=0.032$ ). During the follow-up period, 172 patients (29.5\%) developed MACE. But the combined detection did not predict the long-term clinical outcome.

Conclusion The combination of WCC and TB is an independent predictor for in-hospital outcomes in patients with STEMI than single detection.

\section{Strengths and limitations of this study}

This was the first study to indicate the combined effect of white cell count (WCC) and total bilirubin (TB) on predicting clinical prognosis in patients with acute ST-segment elevation myocardial infarction (STEMI) postprimary percutaneous coronary intervention $(\mathrm{PCl})$.

- The combination of WCC and TB may be better than single biomarker, can be applied in the predicting in-hospital major adverse cardiovascular event of patients with STEMI treated with $\mathrm{PCl}$.

- A single-centre prospective cohort study for a small number of patients was fail to adequately exclude the influence of unknown confounding factors on this study.

- The determination of bilirubin in patients with STEMI was only at a preoperative time point, and the dynamic changes of the bilirubin concentrations during the hospitalisation and follow-up were not clear.

\section{INTRODUCTION}

ST-segment elevation myocardial infarction (STEMI) has been a hot spot in cardiology research with its urgent onset, rapid progress and high mortality. Although reperfusion therapy, such as percutaneous coronary intervention (PCI) and fibrinolytic therapy, had improved the survival rate for acute STEMI, the in-hospital mortality rate of STEMI is as high as $4 \%-12 \%{ }^{1}$ It has been a new challenge for clinician to identify high-risk patients timely, evaluate clinical prognosis accurately and prevent the occurrence of major adverse cardiovascular events (MACE). Screening a sensitive and specific biological index has great value for the treatment and prevention of STEMI complications. 
In an attempt to identify patients with STEMI at high risk of unfavourable outcomes, several predictors of adverse events in STEMI have been investigated. Research indicates that older age, ${ }^{2}$ B-type natriuretic peptide (BNP), D-dimer, uric acid and thrombolysis myocardial infarction (TIMI) risk score are associated with a higher risk of adverse events in patients with STEMI. ${ }^{3-6}$ However, there is no literature report on the combination of white cell count (WCC) and total bilirubin (TB) in the guiding significance for the prognosis of STEMI.

STEMI refers to the occurrence of plaque rupture, thrombosis or coronary artery spasm on the basis of coronary atherosclerosis, which results in a sharp decrease or interruption of coronary artery blood supply and a sustained and severe acute ischaemia of the corresponding myocardium, leading to acute myocardial necrosis. Studies have shown that there are obvious inflammatory reactions and oxidative stress injury in STEMI. ${ }^{78}$ Leucocyte elevation after STEMI is an important component of systemic inflammatory response and ischaemic tissue repair mechanism. Ischaemia-induced chemokines activate WCC to chemotaxis to ischaemic sites and remove necrotic tissues, WCC adhere to the injured vascular wall and form aggregates WCC, which eventually lead to thrombosis. ${ }^{9}$ Activated WCC also produces oxygen-free radicals, lysosomal enzymes and other substances, which cause local inflammatory response in ischaemic sites. ${ }^{10}$ WCC, as a marker of inflammation, has been proved to be closely related to the clinical prognosis of patients with STEMI ${ }^{11}$ while bilirubin, the end product of heme degradation, is also an endogenous oxidant in vivo, participates in the occurrence and development of myocardial infarction. ${ }^{12}$ Heme oxygenase $(\mathrm{HO})$ regulates the synthesis and catabolism of bilirubin, and keeps the bilirubin content in a dynamic balance in human body. ${ }^{13} \mathrm{HO}-1$ is an inducible isoform in response to diverse cellular stress, such as oxidative stress, Inflammatory cytokines, heavy metals, cytokines, but is not expressed under normal conditions. Acute myocardial ischaemia and hypoxia activate the stress process of the body and produces oxygen-free radicals, oxides and infarction-related inflammatory factors, which significantly increase the activity of HO-1, and eventually lead to an increase in bilirubin. Studies have been suggested that bilirubin is elevated in patients with STEMI and has the effect of antioxidant stress, can be used as a biomarker for predicting clinical prognosis. ${ }^{14}$

Thus, there is a strong correlation between inflammatory response and oxidative stress, it is interact and influence each other to promote the development of STEMI. WCC and bilirubin, common and fast acquired biomarkers in routine blood tests, could reflect the level of inflammation and oxidative stress injury, respectively, and that combined information from multiple inflammatory and oxidative stress injury might be more informative. Therefore, the present study first combined WCC (an inflammatory biomarker) and TB (an oxidative stress biomarker) into a simplified equation and assessed whether this value was predictive of in-hospital MACE in patients with STEMI in order to find a simple and reliable auxiliary index to evaluate and predict the clinical prognosis of STEMI for early treatment.

\section{METHODS}

\section{Study design}

This was a single-centre prospective cohort study designed to assess whether the combination of admission WCC and TB could predict in-hospital and long-term outcomes in patients with STEMI postprimary PCI.

We consecutively enrolled adult patients with STEMI who treated with primary PCI at the Cardiac Center of the First Affiliated Hospital of Xinjiang Medical University between November 2012 and June 2017. STEMI diagnosis was in accordance with previously established guidelines. ${ }^{1}$ All patients were treated with PCI within 12 hours after symptoms. Patients who did not have pre-PCI, WCC and bilirubin data, who had severe liver and kidney diseases, autoimmune system diseases, severe heart valve diseases, chronic inflammatory diseases, acute infectious diseases, malignant tumours, blood system diseases, active haemorrhage and other diseases, additionally, patients who had an infarct-related lesion unsuitable for stent implantation, and who were lost to follow-up were excluded from the current study.

\section{Data collection}

Investigators trained by professionals used uniformly designed questionnaires to collect patients' general information including age, gender, body mass index (BMI), history of smoking, dyslipidaemia, hypertension, diabetes, laboratory examination results, angiographic examination results, in-hospital medication and the occurrence of MACE in our hospital by electronic medical records and paper cases.

\section{Blood sampling and haematology measurements}

Blood samples were collected from all participants immediately on hospital admission for complete blood count analyses. In all patients, venous blood samples for laboratory analysis were collected at the time of presentation before the patients were transferred to the heart centre. Peripheral blood samples were used for laboratory tests of several markers, including WCC, total cholesterol, triglycerides, high-density lipoprotein cholesterol (HDLc), low-density lipoprotein cholesterol (LDL-c), TB, direct bilirubin, indirect bilirubin, creatine kinase isoenzyme (CK-MB), platelet, haemoglobin, creatinine and uric acid. $\mathrm{TB}$, direct bilirubin, indirect bilirubin were measured by Hitachi 7060 automatic biochemical analysis and the automatic blood analysis equipment (sysmex XE-5000 automatic blood analyzer) was used for WCC in our hospital testing centre. The normal range for WCC is $(4-10) \times 10^{9} / \mathrm{L}$, TB is $5.5-27.5 \mu \mathrm{mol} / \mathrm{L}$.

All patients were assessed by transthoracic echocardiography within 48 hours after primary PCI standard echocardiographic views were acquired and analysed by two 
experienced cardiologists who were unware of grouping information. Left ventricular ejection fraction (LVEF) was measured by B mode echocardiography.

\section{Definition of cardiovascular risk factors}

Hypertension was defined as self-reported use of antihypertensive medication within the past 2 weeks or having systolic blood pressure (SBP) greater than $140 \mathrm{~mm} \mathrm{Hg}$ and/or diastolic blood pressure (DBP) greater than $90 \mathrm{~mm} \mathrm{Hg}$. Diabetes was defined as fasting blood glucose of more than $7 \mathrm{mmol} / \mathrm{L}$ (or 2 hours after a meal), and/ or, using of insulin or oral hypoglycaemic agents or a selfreported history of diabetes. Hyperlipidaemia was defined as anyone of the four lipids abnormalities following or self-reported use of antihyperlipidaemic medication, total cholesterol concentration $>6.22 \mathrm{mmol} / \mathrm{L}(240 \mathrm{mg}$ / $\mathrm{dL}$ ), triglyceride concentration $>2.26 \mathrm{mmol} / \mathrm{L}$ (200 $\mathrm{mg} /$ $\mathrm{dL}$ ), low-density lipoprotein cholesterol concentration $>4.14 \mathrm{mmol} / \mathrm{L} \quad(160 \mathrm{mg} / \mathrm{dL})$, HDL-C concentration $<1.04 \mathrm{mmol} / \mathrm{L}(40 \mathrm{mg} / \mathrm{dL}){ }^{15}$ Global Registry of Acute Coronary Events (GRACE) score of patients was calculated using eight indicators of GRACE admission risk score according to the medical history, signs and laboratory examination results of admission, including Killip classification of cardiac function, age, heart rate, arterial systolic pressure, serum creatinine, ST segment changes of ECG, elevated myocardial markers, cardiac arrest at admission. Positive smoking history was defined as having smoked daily or occasionally in the past.

\section{PCI and medication}

Patients were given loaded medication before operation: aspirin +clopidogrel (300 mg $+300 \mathrm{mg})$ orally and secondary clinical prophylactic medication after PCI. In our study, angiograms were independently reviewed by two interventional cardiologists who were blinded of patients' information in the Cardiac Center of the First Affiliated Hospital of Xinjiang Medical University. The authors of this article were not involved in the PCI treatment of these patients. At the same time, the results of angiography were recorded for each patient. The main vessel was left main coronary artery, left anterior descending branch, left circumflex branch and right coronary descending branch. The degree of coronary artery stenosis was assessed by cardiovascular computer quantitative analysis system,AXIOM Artis FC/BC, Germany. Cross-sectional projections of right and left coronary arteries were recorded. Most of the approaches were via radial artery, and a few were via femoral artery. There are at least four projection positions for the left coronary artery and at least two projection positions for the right coronary artery. If necessary, other postures are added to fully display each segment of the coronary artery. The imaging data of each patient undergoing coronal angiography are kept for reference. After angiography, more than two experienced interventional physicians including the surgeon discussed the results and filled in the detailed report of the results of coronary angiography after reaching consensus. The number of stenosis branches is defined as a single vessel $>70 \%$ or two vessels more than $50 \%$. PCI was performed by a professional cardiologist, and the stent implantation site, balloon type, pressure, stent size and type, TIMI blood flow classification were filled in detail after the operation.

Successful coronary angiography is defined as residual stenosis $<50 \%$ and thrombolysis in myocardial infarction grade 3 flow after the procedure.

\section{Clinical outcomes and definitions}

In-hospital endpoints: (1) in-hospital mortality; (2) MACE during hospitalisation including cardiac shock, malignant arrhythmia (ventricular tachycardia, ventricular fibrillation), severe cardiac insufficiency, non-fatal myocardial infarction.

Long-term follow-up endpoints: MACE including cardiac death, angina pectoris readmission, non-fatal myocardial infarction, malignant arrhythmia (ventricular tachycardia and ventricular fibrillation), severe cardiac insufficiency (cardiac III-IV level), stent restenosis, target vessels revascularisation. Follow-up was performed by telephone interview for all patients enrolled. For those patients who reached at least one of the primary endpoints, recorded data and medical reports were evaluated to determine inclusion criteria.

\section{Statistical analysis}

Data were collected using EpiData V.3.1 (Odense, Denmark) and double checked. Analyses were carried out using Stata V.15.0 software (Stata). Continuous variables with a Gaussian distribution are presented as average $\pm \mathrm{SD}$, and those with a non-Gaussian distribution are presented as median values with corresponding 25-75th percentiles. The differences between groups were evaluated using Student's unpaired t-test, one-way analysis of variance or the Mann-Whitney rank test. Categorical variables were expressed as numbers and frequencies and the difference between groups was detected by $\mathrm{X}^{2}$ test. Logistic regression was used to determine the combined equation receiver operating characteristic (ROC) curve was used to evaluate the combined value of WCC and TB to predict the best clinical boundary of in-hospital mortality. Univariate and multivariable logistic regression was used to analyse all potential influencing factors associated with in-hospital MACE. ORs are shown with 95\% CIs. The multivariate analysis controlled for all factors with significant associations emerging from the univariate analysis and the traditional risk factors for atherosclerosis. Kaplan-Meier plots were generated, and the log-rank test was used to compare the resulting curves. All statistical tests were bilateral tests, and the significance level was 0.05 .

We included the subjects according to the sample size calculation formula. The sample size calculation formula in this research is $n=2 \overline{p q}\left(u_{\alpha}+u_{\beta}\right)^{2} /\left(p_{1}-p_{0}\right)^{2}$

Based on the sample size of the current study, the power of the research results using power and sample size calculation is $81.2 \%$. 


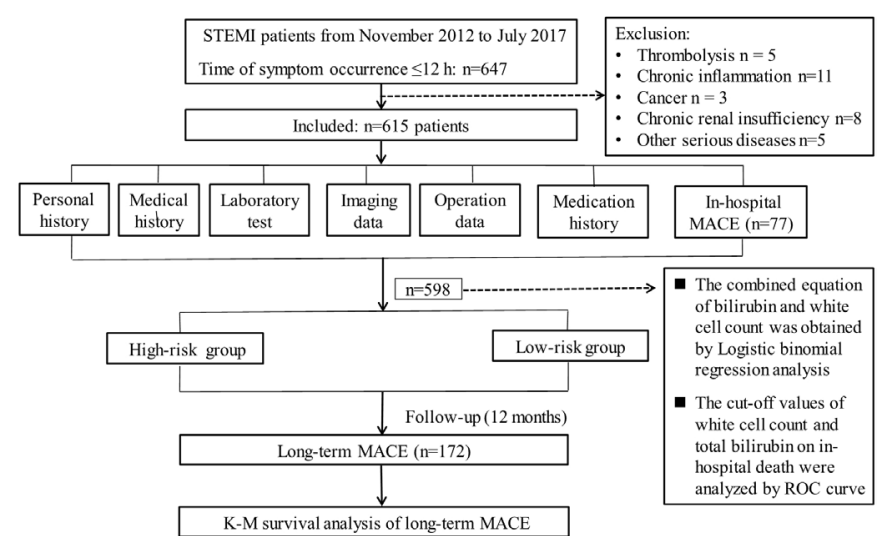

Figure 1 The flowchart of study design with including and excluding procedures. K-M, Kaplan-Meier; MACE, major adverse cardiovascular event; ROC, receiver operating characteristic; STEMI, ST-segment elevation myocardial infarction.

\section{Patients and public involvement}

The study designed to assess whether the combination of admission WCC and TB could predict in-hospital and long-term outcomes in patients with STEMI treated with primary PCI. However, no patients or members of the public were included in the design, recruitment or conduct of the study. The results of measurements would be disseminated to participants after the study, which was completed by the study team. The burden of the intervention will not be assessed by patients themselves.

\section{RESULTS}

\section{Baseline clinical characteristics}

During November 2012 and June 2017, we consecutively recruited 647 adult patients with STEMI who underwent primary PCI. Of these patients, 32 were excluded according to the exclusion criteria. A total of 615 patients with STEMI (525 males and 90 females) with an average age of 58 years were included in this study. In-hospital MACE was found in 77 patients, including 17 patients with in-hospital mortality. Figure 1 depicts the flow chart of the study design. The comparison of the MACE group and non-MACE group are summarised in table 1, including 17 patients with in-hospital mortality (online supplementary table $\mathrm{S} 1$ ).

The patients with MACE were older than without MACE group $(61.2 \pm 11.8$ vs $57.8 \pm 12.2, p=0.019)$. The LVEF in MACE group was worse than that of non-MACE group $(55.48 \pm 7.82$ vs $58.52 \pm 6.58, \mathrm{p}<0.001)$. The laboratory data of the patients showed that the WCC and TB was $(13.01 \pm 4.11) \times 10^{9} / \mathrm{L}$ and $17.21 \pm 6.49 \mathrm{mmol} / \mathrm{L}$ in the MACE group, and $(10.31 \pm 2.85) \times 10^{9} / \mathrm{L}$ and $13.34 \pm 4.91 \mathrm{mmol} / \mathrm{L}$ in the non-MACE group, respectively $(\mathrm{p}<0.001)$. Patients in MACE group also had significantly greater levels of uric acid $(6.67 \pm 2.40$ vs $5.27 \pm 1.70 \mu \mathrm{mol} / \mathrm{L}, \mathrm{p}<0.001)$, creatinine $(83.95 \pm 24.38$ vs $75.46 \pm 18.79 \mu \mathrm{mol} / \mathrm{L}, \mathrm{p}=0.004)$ and greater number of culprit vessels $(3.21 \pm 1.25$ vs $2.80 \pm 1.27$, $\mathrm{p}=0.009)$. The TIMI flow grade after PCI in non-MACE group was higher than MACE group (3(1-3) vs 2 (1-3), $\mathrm{p}=0.023)$. The usage rates of clopidogrel $(92.6 \%$ vs $83.1 \%, \mathrm{p}=0.006)$ and statins $(93.9 \%$ vs $85.7 \%, \mathrm{p}=0.009)$ in the MACE group were lower than those in the non-MACE group.

No significant differences were found between the MACE group and non-MACE group in terms of gender, BMI, smoking, hypertension, diabetes, hyperlipidaemia, SBP, DBP, heart rate, GRACE score, direct bilirubin, indirect bilirubin, total cholesterol, triglycerides, HDL-C, LDL-C, CK-MB, platelet, haemoglobin and culprit vessels location and other drug utilisation rates (table 1).

\section{Ability of WCC and TB to predict short-term clinical outcome}

WCC and TB were combined using logistic regression. Binomial logistic regression analysis was performed with in-hospital mortality as dependent variable and WCC and $\mathrm{TB}$ as independent variables, the logistic regression model was: logit $(\mathrm{P})=-8.00+0.265 \mathrm{WCC}+0.077 \mathrm{~TB}$ (table 2), so the 'combined' value refers to logit $(\mathrm{P})$ in our report. The recommended cut-off value for combined value based on the maximum of Youden's index on the ROC curve was 1.40 and it had $82.4 \%$ sensitivity and $64.7 \%$ specificity in predicting in-hospital mortality. ROC curve was used to determine the predictive value of WCC and TB for the occurrence of in-hospital mortality in patients with STEMI after primary PCI. The optimal cut-off point of WCC was $12.2 \times 10^{9} / \mathrm{L}$ with sensitivity $71 \%$, and specificity $73.1 \%$ (area under the curve, AUC $0.765,95 \%$ CI 0.625 to $0.904)$, the best cut-off value of TB was $14.4 \mathrm{mmol} / \mathrm{L}$ with sensitivity $76.5 \%$ and specificity $62 \%$ (AUC $0.751,95 \%$ CI 0.660 to 0.842 ).

We made a comparison between new predictive model and traditional predictive model based on hypersensitive troponins (hs-TnT). The recommended cut-off value for peak troponins on the ROC curve was $0.87 \mathrm{ug} / \mathrm{mL}$ and it had $85.2 \%$ sensitivity and $77.8 \%$ specificity in predicting in-hospital mortality (AUC $0.894,95 \%$ CI 0.831 to 0.961 ). The combination of WCC and TB was more valuable (AUC $0.804,95 \%$ CI 0.678 to $0.928, \mathrm{p}<0.001$ ) than single WCC and TB but not as valuable as hs-TnT. The AUCs of WCC, TB and logistic regression combined detection was $0.765,0.751$ and 0.804 separately, which showed significant differences $(\mathrm{p}<0.05)$ (table 3, figure 2). Further, the c-statistic was increased from 0.778 to 0.835 when a combination of WCC and TB.

\section{WCC and TB levels predicted clinical outcomes}

Based on the cut-off value of combined for WCC and TB, patients with STEMI were further divided into the highrisk and low-risk groups. The independent risk factors affecting MACE in hospital were analysed by logistic regression analysis. We first performed univariate logistics analysis, and in the multivariable logistic regression, we included a total of 12 indicators. To avoid the influence of traditional factors on the results, we select the traditional risk factors for atherosclerosis, including age, BMI, gender, smoking, hypertension, diabetes and low-density 
Table 1 Comparison between mace group and non-MACE group

\begin{tabular}{|c|c|c|c|c|}
\hline Variables & $\begin{array}{l}\text { Non-MACE } \\
(n=538)\end{array}$ & $\begin{array}{l}\text { MACE } \\
(n=77)\end{array}$ & $\mathrm{T} / \chi^{2}$ & $P$ value \\
\hline Gender (men) (n, \%) & $465(86.4)$ & $63(81.8)$ & 1.180 & 0.277 \\
\hline BMI $\left(\mathrm{kg} / \mathrm{m}^{2}\right)$ & $25.45 \pm 3.29$ & $24.99 \pm 2.90$ & 1.161 & 0.246 \\
\hline Smoking history (n,\%) & $302(56.1)$ & $47(61.0)$ & 0.660 & 0.416 \\
\hline Hyperlipaemia (n,\%) & $396(73.6)$ & $61(79.2)$ & 1.112 & 0.292 \\
\hline $\mathrm{SBP}(\mathrm{mmHg})$ & $121.88 \pm 19.08$ & $118.75 \pm 18.45$ & 1.348 & 0.178 \\
\hline $\mathrm{DBP}(\mathrm{mmHg})$ & $76.58 \pm 13.13$ & $75.18 \pm 13.33$ & 0.870 & 0.385 \\
\hline Heart rate (/min) & $81.76 \pm 13.64$ & $84.69 \pm 17.93$ & 1.686 & 0.092 \\
\hline IBL ( $\mu \mathrm{mol} / \mathrm{L})$ & $11.97 \pm 5.11$ & $11.36 \pm 4.77$ & 0.986 & 0.325 \\
\hline $\mathrm{TC}(\mathrm{mmol} / \mathrm{L})$ & $4.4 \pm 1.18$ & $4.38 \pm 1.18$ & 0.193 & 0.847 \\
\hline $\mathrm{TG}(\mathrm{mmol} / \mathrm{L})$ & $2.05 \pm 1.40$ & $1.93 \pm 1.33$ & 0.734 & 0.463 \\
\hline LDL (mmol/L) & $2.86 \pm 0.85$ & $2.8 \pm 0.84$ & 0.544 & 0.587 \\
\hline HDL (mmol/L) & $1.03 \pm 0.35$ & $1.03 \pm 0.31$ & 0.004 & 0.997 \\
\hline CK-MB (U/L) & $277.73 \pm 221$ & $306.14 \pm 204.18$ & 1.065 & 0.287 \\
\hline $\operatorname{WCC}\left(\times 10^{9} / \mathrm{L}\right)^{\star}$ & $10.31 \pm 2.85$ & $13.01 \pm 4.11$ & 5.569 & $<0.001$ \\
\hline $\mathrm{Hb}(\mathrm{g} / \mathrm{L})$ & $145.24 \pm 19.96$ & $142.47 \pm 23.72$ & 1.114 & 0.266 \\
\hline $\operatorname{LCX}(n, \%)$ & $334(62.1)$ & $53(68.8)$ & 1.315 & 0.251 \\
\hline $\operatorname{RAD}(n, \%)$ & $372(69.1)$ & $61(79.2)$ & 3.282 & 0.070 \\
\hline Culprit Vessels* & $2.80 \pm 1.27$ & $3.21 \pm 1.25$ & 2.631 & 0.009 \\
\hline TIMI grade post-PCl* & $3(1 \sim 3)$ & $2(1 \sim 3)$ & 2.567 & 0.023 \\
\hline \multicolumn{5}{|l|}{ Medication } \\
\hline Aspirin & $519(96.5)$ & $73(94.8)$ & 0.518 & 0.472 \\
\hline Clopidogrel $^{\star}$ & $498(92.6)$ & $64(83.1)$ & 7.635 & 0.006 \\
\hline$\beta$ blockers & $441(82.0)$ & $62(80.5)$ & 0.095 & 0.758 \\
\hline ACEI & $382(71.0)$ & $55(71.4)$ & 0.006 & 0.939 \\
\hline Statins* & 505 (93.9) & $66(85.7)$ & 6.739 & 0.009 \\
\hline
\end{tabular}

${ }^{*} \mathrm{P}<0.05$

$\mathrm{ACEl}$, angiotensin-converting enzyme inhibitor; BMI, body mass index; CKMB, creatine kinase isoenzymes; CR, creatinine; DBL, direct bilirubin; DBP, diastolic blood pressure; GRACE, globla registry of acute coronary events; Hb, haemoglobin; HDL-C, high-density lipoprotein cholesterol; IBL, indirect bilirubin; LAD, left anterior descending branch ; LCX, left circumflex branch; LDL-C, low-density lipoprotein cholesterol; LM, left main coronary artery; LVEF, left ventricular ejection fraction; PCI, percutaneous coronary intervention; PLT, platelet; RAD, right coronary descending branch; SBP, systolic blood pressure; TB, total bilirubin; TC, total cholesterol; TG, triglycerides; TIMI, thrombolysis in myocardial infarction; UC, uric acid; WCC, white cell count. 
Table 2 The combined equation of logistic regression of in-hospital mortality

\begin{tabular}{lrrrr}
\hline Variables & B & OR & \multicolumn{1}{c}{$\mathbf{9 5 \%} \mathbf{C l}$} & P value \\
\hline TB & 0.077 & 1.080 & -0.005 to 0.159 & 0.067 \\
WCC & 0.265 & 1.303 & 0.125 to 0.405 & $<0.001$ \\
Constant term & -8.004 & 0.002 & & $<0.001$ \\
\hline
\end{tabular}

TB, total bilirubin; WCC, white cell count.

lipoprotein cholesterol. We also choose age, diabetes, hypertension, heart rate because they are included in the TIMI risk score, with LVEF, culprit vessels and combine detection, has clinical significance in the univariate analysis. And we choose CK-MB, because CK-MB represents the area of myocardial necrosis and we considered there is a relationship between CK-MB and the poor prognosis of patients. After adjusting, a significant association was noted between combined detection and the adjusted risk of in-hospital MACE (OR 5.85, 95\% CI 3.425 to 9.990, $\mathrm{p}<0.001)$. The results showed that the higher value of equation is an independent predictor for in-hospital MACE of patients with STEMI received primary PCI. Other independent predictors of in-hospital MACE were age (OR $=1.03,95 \%$ CI $1.002 \sim 1.049, \mathrm{p}=0.028)$, LVEF (OR $0.960,95 \%$ CI 0.928 to $0.990, \mathrm{p}=0.010$ ) and number of culprit vessels (OR 1.25, 95\% CI 1.019 to $1.529, \mathrm{p}=0.032$ ) (table 4).

All patients were followed up for an average of 30.2 months, follow-up via telephone was complete in 583 patients or family members, $15(2.5 \%)$ patients were lost to follow-up. After following 172 patients occurred in MACE with the incidence of out-hospital MACE was 29.5\%. Kaplan-Meier survival analysis of long-term MACE was made by dividing the best truncation value into two groups. The results showed that there was no significant difference in the long-term incidence of MACE between the two groups ( $\mathrm{p}=0.869$, figure 3 ).

\section{DISCUSSION}

The current study is the first to indicate that the combined effect of WCC and TB could better predict prognosis (in terms of in-hospital mortality and in-hospital MACE) in patients with acute STEMI undergoing PCI compared with single predictor independently. Our

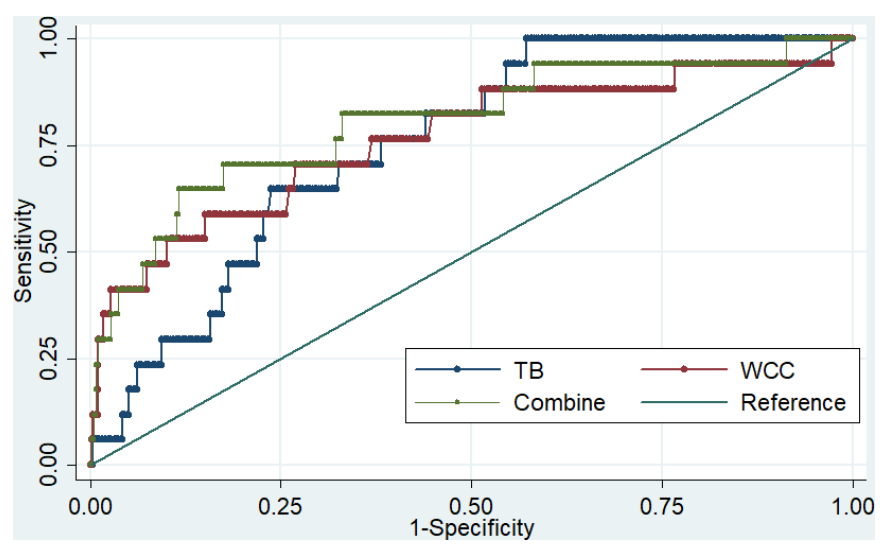

Figure 2 Admission of TB, WCC and combine as predictors of in-hospital mortality. TB, total bilirubin; WCC, white cell count.

studies suggesting that those with higher WCC and TB had a higher likelihood of in-hospital complications, and a higher hazard of in-hospital mortality, independent of potential confounding factors.

Inflammatory processes play an important role in the development of STEMI. It has been suggested that there is a positive correlation between leucocyte elevation and myocardial infarction area. ${ }^{16-18}$ WCC in the unstable plaque lesions adhesion, aggregation and release the tissue factor and promote blood clotting enzyme molecules, prompt production platelet activation and fibrin thrombi. When great quantities of WCG infiltrate in the ischaemic area, because of its weak deformation, slow through the capillary, capillaries are more likely to block the ischaemia area, increase myocardial microcirculation, led to a lack of oxygen to microvascular no reflow and aggravate ischaemic infarction area. ${ }^{16} 19$ The increase of WCC count has an impact on the occurrence, development and prognosis of acute myocardial infarction (AMI), and the relevant mechanism is generally believed that the capillary is blocked by a large number of WCC, which causes no reflow of microvessels and increases the size of myocardial infarction..$^{20}$ Studies have proposed a positive correlation between leucocyte elevation and myocardial infarction area. ${ }^{21}$

There is indeed a lot of literature on the relationship between leucocytes and prognosis of ischaemic heart disease. Kyne suggested that the increase of WCC count and neutrophilic granulocyte percentage in peripheral

Table 3 Admission of TB, WCC and combine as predictors of in-hospital mortality

\begin{tabular}{|c|c|c|c|c|c|c|}
\hline Variables & Sensitivity (\%) & $\begin{array}{l}\text { Specificity } \\
(\%)\end{array}$ & $\begin{array}{l}\text { AUC } \\
(0 \text { to 1.0) }\end{array}$ & $95 \% \mathrm{Cl}$ & C-statistic & $P$ value \\
\hline TB ( $\geq 14.4 \mathrm{mmol} / \mathrm{L})$ & 76.5 & 62.0 & 0.751 & 0.660 to 0.842 & 7.989 & 0.032 \\
\hline Combine $(\geq 1.4)$ & 82.4 & 64.7 & 0.804 & 0.678 to 0.928 & & \\
\hline hs-TnT ( $\geq 0.87 \mu \mathrm{g} / \mathrm{mL})$ & 85.2 & 77.8 & 0.894 & 0.831 to 0.961 & & \\
\hline
\end{tabular}

AUC, area under the curve; hs-TnT, hypersensitive troponin; TB, total bilirubin; WCC, white cell count. 
Table 4 Univariate and multivariate logistic analysis of in-hospital mace

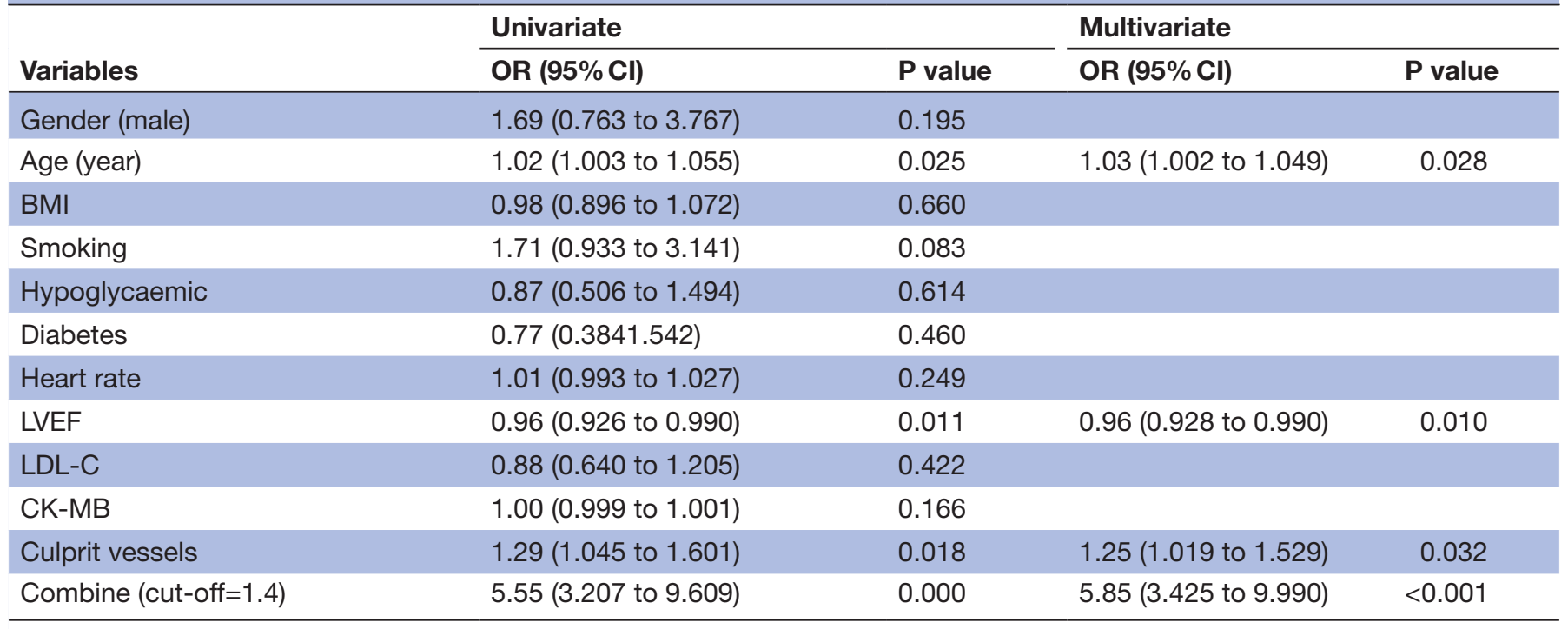

BMI, body mass index; CK-MB, creatine kinase isoenzymes; LDL-C, low-density lipoprotein cholesterol; LVEF, left ventricular ejection fraction.

blood within 12 hours was an independent predictor of heart failure in the first 4 days after AMI. ${ }^{22}$ Kojima et al demonstrated an elevated WCC was significantly associated with higher risk of in-hospital mortality. ${ }^{23}$ Nunez reported that WCC independently increased the risk of death for 30 days and 1 year in patients with STEMI. ${ }^{24}$ Ciçek et al examined that the incidence of MACE in atients with STEMI with elevated WCC increased significantly during the hospitalisation. ${ }^{25}$

This means that leucocyte elevation during myocardial infarction has a purely restorative physiological role, and has a pathological role. Combined with myocardial infarction size, non-coronary reflux and decreased cardiac function, increased WCC in patients with STEMI may indicate poor clinical prognosis.

In addition to the inflammatory, oxidative stress injury plays an important role in the evolution of STEMI. ${ }^{26}$ Under physiological conditions, antioxidant substances in the body are in equilibrium with oxygen-free radicals.

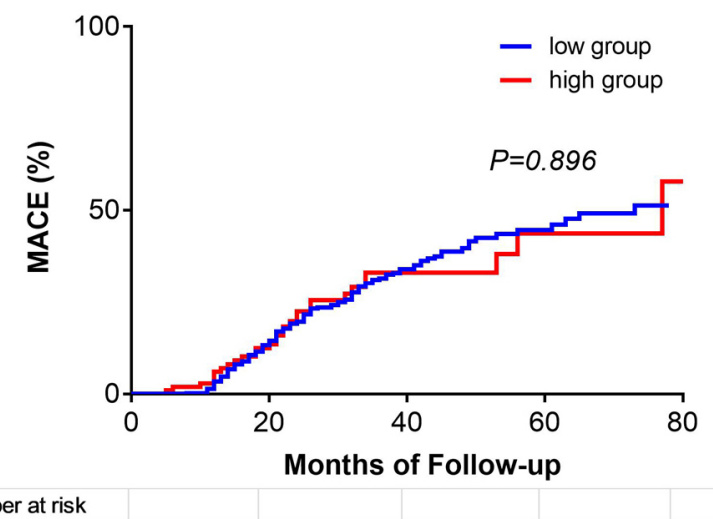

\begin{tabular}{|c|c|c|c|c|c|}
\hline Number at risk & & & & & \\
\hline Low group & 505 & 353 & 127 & 42 & 0 \\
\hline High group & 110 & 76 & 27 & 8 & 1 \\
\hline
\end{tabular}

Figure 3 Kaplan-Meier survival analysis of long-term mace. MACE, major adverse cardiovascular event.
When STEMI occurs, the surface of coronary artery intima and lipid plaques are damaged. Plaque rupture and thrombosis lead to acute myocardial ischaemia and hypoxia, activates the stress process cells and cytoplasm produce large amounts of oxygen-free radicals through enzymatic and non-enzymatic systems, which lead to the oxidation of macromolecular protein, cell membrane lipid peroxidation and DNA damage, and finally cause the death of heart muscle cells. Myocardial cells after STEMI are stimulated by ischaemia and hypoxia to produce large amounts of oxygen-free radicals and oxidising substances, a large amount of antioxidant substances are consumed so that the body loses its inhibiting effect on the formation of oxidative substances, and the balance between antioxidant substances and oxidative substances is broken, which will cause cell structure damage. ${ }^{27}$ Oxidative stress injury can cause plaque instability, rupture and erosion, leading to thrombosis and complete infarctionrelated artery occlusion. ${ }^{28}$ So when STEMI occurs, the body needs antioxidants to deal with the damage caused by oxidative stress.

Antioxidants and markers which can represent oxidative reactions in vivo have been reported in the previous literature. Malondialdehyde is the metabolite of unsaturated fatty acids in biofilms after oxidative damage, while advanced oxidation protein product is the metabolite of proteins after free radical attack and oxidative reaction, which can reflect the degree of oxidative reaction. ${ }^{29}$ Superoxide dismutase (SOD) and glutathione (GSH), as important antioxidant substances in the body, can remove excess oxygen-free radicals in the body and maintain the reduced state of cells. ${ }^{30}$ When acute coronary syndrome occurs, GSH and SOD in serum are reduced. ${ }^{31}{ }^{32}$ Previous studies have reported impaired oxidation and antioxidant balance and increased oxidative stress in patients with STEMI. ${ }^{33}$ Gür et al showed that total antioxidant 
capacity levels were significantly lower and total oxidation state, oxidative stress index, lipid hydroperoxide were significantly higher in patients with no-reflow compared with normal flow group in patients with anterior STEMI undergoing primary PCI. ${ }^{8}$ Serdar et al showed that in the development process from unstable angina to STEMI, total sialic acid, oxidative modified protein and other oxidation substances were gradually increased, and the antioxidant status was gradually decreased. ${ }^{34}$ These oxidation substances were more significant in patients with STEMI. However, these substances require a certain amount of technology and time to test, and not all hospitals have the capability.

Bilirubin is an endogenous oxidant in vivo. In recent years, more and more attention has been paid to the role of bilirubin in the pathophysiology of major cardiovascular diseases such as myocardial infarction, ${ }^{35}$ atherosclerosis $^{36}$ and cardiovascular complications of diabetes. ${ }^{37}$ As an antioxidant in the body, bilirubin is involved in the occurrence and development of myocardial infarction and plays an antioxidative role. Okuhara et al showed that compared with non-AMI patients, the serum bilirubin concentration and $\mathrm{Fe}^{2}$ in $\mathrm{AMI}$ patients increased temporarily 18-21 hours after the onset of the disease. ${ }^{12}$ Elevated bilirubin has antioxidant capacity and the ability to remove peroxides, which can prevent the deterioration of disease. Sexual resistance to oxidative stress in the myocardium may be considered as a beneficial selfregulation in acute ischaemia. Celik et al reported that TB levels in STEMI were independently associated with no-reflow coronary artery. ${ }^{38}$ Halit et al reported that bilirubin levels in patients with STEMI with impaired blood flow were higher than those in normal blood flow group. They suggested that the more severe the atherosclerosis, the higher the activity of HO-1 enzyme after myocardial infarction, the more obvious the increase of bilirubin levels. ${ }^{39}$ The degree of increase was related to the severity of the lesion to a certain extent. Gul et al studied 1624 patients with STEMI and found that compared with the bilirubin group less than $9.0 \mathrm{mg} / \mathrm{dL}$, the bilirubin group higher than $9.0 \mathrm{mg} / \mathrm{dL}$ had higher in-hospital mortality, but the bilirubin level was not related to long-term prognosis. ${ }^{40}$ Chung et al followed up 1111 patients with STEMI treated with PCI in hospital and 12 months after operation. The results showed that the incidence of MACE and cardiogenic mortality in hyperbilirubinaemia group were higher than those in low bilirubinaemia group. ${ }^{14}$ It can be concluded that the oxygen-free radicals produced by oxidative stress injury and the damage of various oxides to the body under stress may exceed the protective and antioxidant effect of bilirubin on the body, which caused the protective effect of high bilirubin level is not significant. In contrast to stable coronary disease, the serum TB levels show different associations in stressful conditions

Therefore, STEMI is a disease both with inflammatory and oxidative stress injury. As such, inflammatory biomarkers alone or oxidative stress are not sufficient to capture the entire pathophysiological process involved in
STEMI. Instead, markers which combine inflammatory and oxidative stress information is a better at reflecting the process in STEMI.

In the present study, the area under the ROC curve for combined equation in predicting mortality was higher than those for WCC and TB, multivariate logistic regression analysis showed the high value of equation is an independent predicted of worse in-hospital MACE. In addition to the elevation of WCC and bilirubin in the MACE group, TIMI blood flow was also worse than that in the non-MACE group. Integrate previous studies, we think the increase of WCC count causes no reflow of microvessels and increases the size of myocardial infarction and the increase of TB levels reflect the severity of coronary damage, which suggests that combining WCC and $\mathrm{TB}$ has a stronger predictive power for in-hospital MACE than individual markers. Previous studies have shown that the in-hospital mortality of STEMI in China between 2001 and 2011 was $7.0 \%{ }^{41}$ In our study, in-hospital MACE was found in $77(12.5 \%)$ patients and there were $17(2.8 \%)$ patients occurred in death in-hospital among the 615 patients with STEMI, the in-hospital mortality was comparable to the China's national level, so we think our results are reliable. We did not use troponin levels and other biomarkers that are recommended generally to predict clinical outcomes. As we know, troponin test fee will be more expensive and testing requirements for basic-level hospitals is too high, not all hospitals have this condition, while WCC and bilirubin, common and fast acquired biomarkers in routine blood tests, can be detected by most hospitals because of low test cost . So we still suggested that combining WCC and TB was of particular clinical importance for the subset of patients with STEMI at admission.

There are some limitations in our study. This study is a single-centre experiment for a few patients which have the limitation of prospective cohort design. In our study, bilirubin was measured in patients at only one time point before PCI in this study. The dynamic changes of serum leucocyte count and bilirubin concentration during the hospitalisation and follow-up were not clear. In this study, oxidative stress-related indicators and HO-1 enzyme activity were not directly tested, and various inflammatory markers such as $\mathrm{C}$ reactive protein, neutrophils and lymphocytes were not included. In addition to determine the exact role of leucocytes and bilirubin in patients for long-term prognosis more experimental basic studies are needed.

\section{CONCLUSIONS}

The combination of WCC and TB is better than single biomarker, can be applied in the predicting in-hospital MACE of patients with STEMI treated with primary PCI. The high value of equation is a independent predictor of worse in-hospital MACE, it can be used to identify highrisk patients with STEMI and accurately predict their clinical prognosis for early treatment. 
Author affiliations

${ }^{1}$ Department of Cardiology, First Affiliated Hospital of Xinjiang Medical University, Urumqi, China

${ }^{2}$ State Key Laboratory of Pathogenesis, Prevention and Treatment of High Incidence Diseases in Central Asian, Urumqi, China

${ }^{3}$ Xinjiang Key Laboratory of Cardiovascular Disease Research, Clinical Medical Research Institute of First Affiliated Hospital of Xinjiang Medical University, Urumqi, China

Acknowledgements The authors would like to thank the Project on Science and Technology Program of Xinjiang Uyghur Autonomous Region and the project grants of the National Natural Science Foundation of China.

Contributors Conceived and designed the experiments: X-ML, Y-NY. Analysed the data: QZ, FL. Contributed reagents/materials/analysis tools: CFS, XRZ, NS, WA, XZ and AG. Quality control the study and revision: X-ML, Y-NY and XY. Wrote the paper: TM and QZ. All authors read and approved the final manuscript.

Funding This work was supported by a project grant from Science and Technology Program of Xinjiang Uyghur Autonomous Region, China (no.2018D04029) and project grants of the National Natural Science Foundation of China (no.81660058).

Competing interests None declared.

Patient consent for publication Not required.

Ethics approval The study complied with the Declaration of Helsinki, the study protocol was first approved in December 2014 by the Ethics Committee of the First Affiliated Hospital of Xinjiang Medical University (Xinjiang, China) (approval ID: 20141201-03) and ethics review was conducted in January 2017 (approval ID:20141201-03-1701A).

\section{Provenance and peer review Not commissioned; externally peer reviewed.}

Data availability statement All data relevant to the study are included in the article and are not publicly available

Open access This is an open access article distributed in accordance with the Creative Commons Attribution Non Commercial (CC BY-NC 4.0) license, which permits others to distribute, remix, adapt, build upon this work non-commercially, and license their derivative works on different terms, provided the original work is properly cited, appropriate credit is given, any changes made indicated, and the use is non-commercial. See: http://creativecommons.org/licenses/by-nc/4.0/.

\section{ORCID iD}

Xiao-Mei Li http://orcid.org/0000-0002-8770-3838

\section{REFERENCES}

1 Arslan F, Bongartz L, Ten Berg JM, et al. 2017 ESC guidelines for the management of acute myocardial infarction in patients presenting with ST-segment elevation: comments from the Dutch ACS Working group. Neth Heart J 2018;26:417-21.

2 de Labriolle A, Pacouret G, Giraudeau B, et al. Effect of time to treatment and age on one year mortality in acute STEMI: difference between thrombolysis and primary percutaneous coronary intervention. Arch Cardiovasc Dis 2008;101:48-54

3 Akgul $\mathrm{O}$, Uyarel $\mathrm{H}$, Pusuroglu $\mathrm{H}$, et al. High BNP level as risk factor for acute kidney injury and predictor of all-cause mortality in STEM patients. Herz 2014;39:507-14.

4 Akgul O, Uyarel $\mathrm{H}$, Pusuroglu $\mathrm{H}$, et al. Predictive value of elevated D-dimer in patients undergoing primary angioplasty for ST elevation myocardial infarction. Blood Coagul Fibrinolysis 2013;24:704-10.

5 Kaya MG, Uyarel H, Akpek M, et al. Prognostic value of uric acid in patients with ST-elevated myocardial infarction undergoing primary coronary intervention. Am J Cardiol 2012;109:486-91.

6 Gevaert SA, De Bacquer D, Evrard P, et al. Gender, TIMI risk score and in-hospital mortality in STEMI patients undergoing primary $\mathrm{PCl}$ : results from the Belgian STEMI registry. Eurolntervention 2014;9:1095-101.

7 Ç Y, Gayretli YK, Açar B, et al. White blood cell subtypes and ratios in cardiovascular disease. Angiology 2016;68:651.

8 Gür M, Türkoğlu C, Tașkın A, et al. Paraoxonase-1 activity and oxidative stress in patients with anterior ST elevation myocardial infarction undergoing primary percutaneous coronary intervention with and without no-reflow. Atherosclerosis 2014;234:415-20.

9 Barron HV, Cannon CP, Murphy SA, et al. Association between white blood cell count, epicardial blood flow, myocardial perfusion, and clinical outcomes in the setting of acute myocardial infarction: a thrombolysis in myocardial infarction 10 substudy. Circulation 2000;102:2329-34

10 Lucchesi B, Werns SW, Fantone JC. The role of the neutrophil and free radicals in ischemic myocardial injury. $\mathrm{J} \mathrm{Mol} \mathrm{Cell} \mathrm{Cardiol}$ 1989;21:1241-51.

11 He R, Li H-yan, Guo L-jun, et al. Predictive value of postprocedural leukocyte count on myocardial perfusion, left ventricular function and clinical outcomes in ST-elevated myocardial infarction after percutaneous coronary intervention. Chin Med J 2012;125:1023-9.

12 Okuhara K, Kisaka T, Ozono R, et al. Change in bilirubin level following acute myocardial infarction is an index for heme oxygenase activation. South Med J 2010;103:876-81.

13 Ayer A, Zarjou A, Agarwal A, et al. Heme oxygenases in cardiovascular health and disease. Physiol Rev 2016;96:1449-508.

14 Chung S-R, Yang T-H, Shin $\mathrm{H}-\mathrm{C}$, et al. Initial total bilirubin and clinica outcome in patients with ST-segment elevation myocardial infarction undergoing primary percutaneous coronary intervention with drugeluting stents. Circ J 2016;80:1437-44.

15 Pan S, Yu Z-X, Ma Y-T, et al. Appropriate body mass index and waist circumference cutoffs for categorization of overweight and central adiposity among Uighur adults in Xinjiang. PLoS One 2013;8:e80185.

16 Swirski FK, Nahrendorf M. Leukocyte behavior in atherosclerosis, myocardial infarction, and heart failure. Science 2013;339:161-6.

17 Chia S, Nagurney JT, Brown DFM, et al. Association of leukocyte and neutrophil counts with infarct size, left ventricular function and outcomes after percutaneous coronary intervention for ST-elevation myocardial infarction. Am J Cardiol 2009;103:333-7.

18 Palmerini T, Mehran R, Dangas G, et al. Impact of leukocyte count on mortality and bleeding in patients with myocardial infarction undergoing primary percutaneous coronary interventions: analysis from the Harmonizing outcome with revascularization and stent in acute myocardial infarction trial. Circulation 2011;123:2829-37.

19 Sager HB, Nahrendorf M. Inflammation: a trigger for acute coronary syndrome. Q J Nucl Med Mol Imaging 2016;60:185-93.

20 Pellizzon GG, Dixon SR, Stone GW, et al. Relation of admission white blood cell count to long-term outcomes after primary coronary angioplasty for acute myocardial infarction (the stent PAMI trial). Am J Cardiol 2003;91:729-31.

21 Husser O, Bodi V, Sanchis J, et al. White blood cell subtypes after STEMI: temporal evolution, association with cardiovascular magnetic Resonance-Derived infarct size and impact on outcome. Inflammation 2011;34:73-84.

22 Kyne L, Hausdorff JM, Knight E, et al. Neutrophilia and congestive heart failure after acute myocardial infarction. Am Heart $J$ 2000;139:94-100.

23 Kojima S, Sakamoto T, Ishihara M, et al. The white blood cell count is an independent predictor of no-reflow and mortality following acute myocardial infarction in the coronary interventional era. Ann Med 2004;36:153-60.

24 Nunez JE. Prognostic value of baseline white blood cell count in patients with acute myocardial infarction and ST segment elevation. Heart 2005:91:1094-5.

25 Çiçek G, Açıkgöz SK, Yayla Çağrı, et al. White blood cell count to mean platelet volume ratio: a novel and promising prognostic marker for ST-segment elevation myocardial infarction. Cardiol $J$ 2016;23:225-35.

26 Fearon IM, Faux SP. Oxidative stress and cardiovascular disease: novel tools give (free) radical insight. J Mol Cell Cardiol 2009;47:372-81.

27 Liang D, Zhang Q, Yang $\mathrm{H}$, et al. Anti-Oxidative stress effect of Loading-Dose rosuvastatin prior to percutaneous coronary intervention in patients with acute coronary syndrome: a prospective randomized controlled clinical trial. Clin Drug Investig 2014;34:773-81.

28 Börekçi A, Gür M, Türkoğlu C, et al. Oxidative stress and spontaneous reperfusion of Infarct-Related artery in patients with STsegment elevation myocardial infarction. Clin Appl Thromb Hemost 2016;22:171-7.

29 Baraas F, Rilantono L, Diniharini S, et al. Effect of short-term lowintensity exercise training on association of oxygen free radicals and nitric oxide production in patients with acute myocardial infarction. Int J Angiol 2013;22:159-64.

30 Matysiak R, Błaszczyk J, Obrebska A, et al. [Oxidative-reductive balance in patients after acute coronary syndrome that undergo cardiac rehabilitation]. Pol Merkur Lekarski 2014;37:148-52.

31 Awadallah S, Al Arrayed K, Bahareth E, et al. Total antioxidant capacity and ischemia modified albumin in beta thalassemia. Clin Lab 2013:59:687-91.

32 Ellidag HY, Eren E, Yılmaz N, et al. Oxidative stress and ischemiamodified albumin in chronic ischemic heart failure. Redox Rep 2014;19:118-23. 
33 LoPresti R, Catania A, D'Amico T, et al. Oxidative stress in young subjects with acute myocardial infarction: evaluation at the initial stage and after 12 months. Clin Appl Thromb Hemost 2008;14:421-7.

34 Serdar Z, Serdar A, Altin A, et al. The relation between oxidant and antioxidant parameters and severity of acute coronary syndromes. Acta Cardiol 2007;62:373-80.

35 Wei S, Mao L, Liu B, et al. Serum biomarkers and the prognosis of AMI patients. Herz 2014;39:384-9.

36 Erkan A, Ekici B, Uğurlu M, et al. The role of bilirubin and its protective function against coronary heart disease. Herz 2014;39:711-5.

37 Vítek L. The role of bilirubin in diabetes, metabolic syndrome, and cardiovascular diseases. Front Pharmacol 2012;3:55.
38 Celik T, Kaya MG, Akpek M, et al. Does serum bilirubin level on admission predict TIMI flow grade and in-hospital mace in patients with STEMI undergoing primary PCl. Angiology 2014;65:198-204.

39 Acet $\mathrm{H}$, Erts $\mathrm{F}$, Akıl MA, et al. A novel predictor of infarct-related artery patency before percutaneous intervention and in-hospital outcomes for ST-segment elevation myocardial infarction patients: serum bilirubin level. Postepy Kardiol Interwencyjnej 2014;10:91-7.

40 Gul M, Uyarel H, Ergelen M, et al. Prognostic value of total bilirubin in patients with ST-Elevated myocardial infarction undergoing primary coronary intervention. J Am Coll Cardiol 2013;62:C20-1.

41 Li J, Li X, Wang Q, et al. St-Segment elevation myocardial infarction in China from 2001 to 2011 (the China PEACE-Retrospective acute myocardial infarction study): a retrospective analysis of hospital data. The Lancet 2015;385:441-51. 